\title{
Sistema de Classificação de Pacientes Pediátricos: construção e validação de categorias de cuidados*
}

\author{
PEDIATRIC PATIENT CLASSIFICATION SYSTEM: CONSTRUCTION AND \\ VALIDATION OF CARE CATEGORIES
}

SISTEMA DE CLASIFICACIÓN DE PACIENTES PEDIÁTRICOS: CONSTRUCCIÓN Y
VALIDACIÓN DE CATEGORIAS DE ATENCIÓN

\begin{abstract}
Ariane Polidoro Dini ${ }^{1}$, Fernanda Maria Togeiro Fugulin², Maria De La Ó Ramallo Veríssimo ${ }^{3}$, Edinêis de Brito Guirardello ${ }^{4}$
\end{abstract}

\section{RESUMO}

A classificação de pacientes é essencial para o gerenciamento de uma unidade. Entretanto, a literatura não dispõe de conceituações de categorias de pacientes pediátricos. Os objetivos deste estudo foram definir e validar categorias de cuidado de pacientes pediátricos, de acordo com o grau de dependência da equipe de enfermagem. Utilizou-se revisão bibliográfica para definição das categorias de cuidado e a validação de conteúdo das categorias propostas foi realizada por um grupo de juízes envolvidos em assistência e gerência de unidades pediátricas e por juízes envolvidos em ensino e pesquisa de pediatria e construção de instrumentos de classificação de pacientes. Foram estabelecidas e validadas cinco categorias de cuidados: Mínimos, Intermediários, Alta dependência, Semi-intensivo e Intensivo. A validação das categorias de cuidado subsidiou a construção de um sistema de classificação de pacientes pediátricos que poderá contribuir para o processo de tomada de decisão do enfermeiro na prática gerencial e assistencial.

\section{DESCRITORES}

Enfermagem pediátrica

Carga de trabalho

Avaliação em enfermagem

Classificação

Estudos de validação

\begin{abstract}
Patient classification is essential for managing a hospital unit. Literature, however, does not present conceptualizations of pediatric patient categories. The objectives of this study were to define and validate pediatric patient care categories, according to the level of dependency of the nursing team. The care categories were defined based on a literature review, and the content of the proposed categories was validated by a group of judges involved in managing and providing care in a pediatric unit, and by judges involved in pediatric education and research as well as in the construction of patient instruments. Five care categories were established and validated: Minimal, Intermediate, High dependency, Semi-intensive and Intensive. The validation of the care categories were the basis for constructing a system for the classification of pediatric patients, that may contribute with the decision making process of nurses working in management and health care.
\end{abstract}

DESCRIPTORS
Pediatrics nursing
Workload
Nursing assessment
Classification
Validation studies

\begin{abstract}
RESUMEN
La clasificación de pacientes es esencial para el gerenciamiento de una unidad. Sin embargo, la literatura no dispone de conceptualizaciones de categorías de pacientes pediátricos. Este estudio objetivó definir y validar categorías de atención de pacientes pediátricos, de acuerdo con grado de dependencia del equipo de enfermería. Se utilizó revisión bibliográfica para definición de categorías de atención y la validación de las categorías propuestas fue efectuada por un grupo de jueces involucrados en atención y gerenciamiento de unidades pediátricas, y por jueces identificados con enseñanza e investigación en pediatría y construcción de instrumentos de clasificación de pacientes. Se establecieron y validaron cinco categorías de atención: Mínimos, Intermedios, Alta Dependencia, Semi-intensivo e Intensivo. La validación de categorías de atención ayudó a construir un sistema de clasificación de pacientes pediátricos que podrá contribuir al proceso de toma de decisiones del enfermero en la práctica gerencial y asistencial.
\end{abstract}

\author{
DESCRIPTORES \\ Enfermería pediátrica \\ Carga de trabajo \\ Evaluación en enfermería \\ Clasificación \\ Estudios de validación
} * Extraído da dissertação "Sistema de Classificação de Pacientes Pediátricos: construção e validação de instrumento", Pós Graduação em Enfermagem da
Faculdade de Ciências Médicas da Universidade Estadual de Campinas, 2008. ${ }^{1}$ Mestre em Enfermagem. Doutoranda de Enfermagem do Departamento de
Enfermagem da Faculdade de Ciências Médicas da Universidade Estadual de Campinas. Enfermeira do Serviço de Enfermagem Pediátrica do Hospital de
Clínicas da Universidade Estadual de Campinas. Campinas, SP, Brasil. aridini@fcm.unicamp.br ${ }^{2}$ Professora Doutora do Departamento de Orientação Profissi-
onal da Escola de Enfermagem da Universidade de São Paulo. São Paulo, SP, Brasil. ffugulim@usp.br ${ }^{3}$ Professora Doutora do Departamento de Enfermagem
Materno-Infantil e Psiquiatria da Escola de Enfermagem da Universidade de São Paulo. São Paulo, SP, Brasil. mdlorver@usp.br ${ }^{4}$ Professora Associada do
Departamento de Enfermagem da Faculdade de Ciências Médicas da Universidade Estadual de Campinas. Campinas, SP, Brasil. guirar@fcm.unicamp.br 


\section{INTRODUÇÃO}

Os serviços de saúde têm como principal objetivo a produção de impacto positivo na condição de saúde da população e, para a organização dos mesmos, é necessária a análise de questões como demanda, oferta e qualidade desses serviços ${ }^{(1)}$.

A qualidade dos serviços de saúde é determinada pelo conjunto da estrutura, do processo de trabalho e dos resultados das ações realizadas para melhorar a saúde da clientela ${ }^{(2)}$. Os serviços de saúde com estruturas apropriadas como área física, recursos humanos e materiais adequados, favorecem condições para prestar uma assistência de melhor qualidade.

O serviço de enfermagem representa papel fundamental no processo assistencial e, na análise da oferta dos serviços de saúde, o gerenciamento de recursos humanos deve receber especial atenção para equilibrar questões como custo, eficácia e qualidade da assistência ${ }^{(3)}$.

Os aspectos quantitativos e qualitativos dos recursos humanos em enfermagem estão diretamente ligados à qualidade da assistência oferecida ao paciente, de forma que requerem a atenção das gerências devido aos reflexos negativos que o dimensionamento inadequado desses recursos pode causar à assistência prestada à clientela ${ }^{(4)}$.

O dimensionamento de pessoal é um processo sistemático que fundamenta o planejamento e a avaliação do quantitativo e qualitativo de pessoal de enfermagem, necessário para prover cuidados de forma a garantir a qualidade, previamente estabelecida, a um grupo de pacientes de acordo com a filosofia, singularidade e estrutura de cada serviço ${ }^{(5)}$.

Para o dimensionamento de pessoal em enfermagem, é necessário realizar o diagnóstico situacional de cada unidade ou serviço, o qual envolve a caracterização da clientela, a filosofia, os objetivos e as propostas assistenciais de cada instituição $0^{(5-8)}$.

Destaca-se que apenas o conhecimento do número de leitos ou o percentual de leitos ocupados não são indicadores seguros para quantificar a demanda de assistência de uma unidade de internação(9-11) , e a caracterização da clientela não se reduz à análise isolada de dados epidemiológicos como idade, sexo e diagnóstico médico. Outrossim, a demanda de cuidados é compreendida pela avaliação do tempo de assistência, dependência para realização de atividades de vida diária, complexidade e severidade da doença ${ }^{(6)}$.

Nesse sentido, a utilização de Sistema de Classificação de Paciente (SCP) permite estimar, quantificar e avaliar a demanda de cuidados de enfermagem por grupos de paci- entes, categorizando-os de acordo com a necessidade de cuidados em um período de tempo específico ${ }^{(9-11)}$. A aplicação de SCP possibilita fundamentar o dimensionamento e a distribuição de pessoal, subsidiando, também, o planejamento e a previsão dos custos, tendo em vista o alcance dos padrões de qualidade da assistência ${ }^{(11)}$, pois permite caracterizar a clientela discriminando diferentes categorias de cuidado, segundo o grau de dependência da equipe de enfermagem.

O Conselho Federal de Enfermagem ${ }^{(8)}$, baseado em estudo nacional(7), estabeleceu parâmetros oficiais para o dimensionamento de enfermagem fundamentando-se na distribuição em categorias de cuidado para indicar o número mínimo de profissionais de enfermagem, bem como a porcentagem de distribuição por categoria profissional ${ }^{(8)}$. As conceituações para as categorias de cuidado do estudo de referência(7) não são adequadas à realidade da pediatria, uma vez que não contemplam o perfil e as necessidades dos pacientes pediátricos e, desta forma, com a lacuna literária de conceituações específicas, a área de pediatria encontra dificuldade para fundamentar seu dimensionamento de pessoal.

Face a esta realidade, faz-se necessário a realização de estudos que conceituem as categorias de cuidado em pediatria.

\section{OBJETIVOS}

Construir e validar a conceituação das categorias de cuidado para a clientela da Enfermagem Pediátrica que distingam diferentes grupos de pacientes em relação às necessidades de cuidado de enfermagem.

\section{MÉTODO}

Realizou-se estudo metodológico, o qual objetivou tornar construtos intangíveis, como fenômenos de interesse para a pesquisa e prática de enfermagem, em tangíveis. Os procedimentos para esse tipo de estudo dependem do uso do construto, porém, a revisão de literatura e a avaliação da validade do mesmo são indispensáveis ${ }^{(12)}$.

A primeira fase do trabalho voltou-se ao delineamento das categorias de cuidado com base em estudo bibliográfico sobre desenvolvimento infantil e conceituações existentes para pacientes adultos.

A segunda fase envolveu a avaliação da validade de conteúdo das conceituações das categorias de cuidado, a qual consiste em verificar o grau de correlação entre o conceito e a medida futura do mesmo ${ }^{(12)}$. No caso deste estudo, consistiu em verificar se as categorias de cuidado distinguem grupos de pacientes, com características comuns, em relação às necessidades de cuidados de enfermagem. 
Para avaliação da validade de conteúdo, utilizou-se a técnica Delphi, que consiste na realização de julgamentos por um grupo de juízes, especialistas na área, por meio de questionários, e, após a resposta de todos os juízes, agrupamento e análise minuciosa das opiniões para aprimorar a idéia inicial e realizar uma nova fase de questionamentos com os mesmos juízes. Podem ser realizadas várias fases de questionamento, seguidas de modificações do constructo, de forma a aperfeiçoá-lo, até que se obtenha o consenso entre o grupo de juízes. Esta técnica é caracterizada pela flexibilidade, onde o pesquisador responsável do estudo estabelece as regras quanto ao número de fases, número de especialistas e o nível de consenso para considerar o construto válido ${ }^{(12-14)}$.

As conceituações das categorias de cuidado foram submetidas à apreciação de um grupo de juízes, composto por enfermeiros que atuam no ensino, na pesquisa, na gerência ou na assistência direta ao paciente pediátrico ou, ain$\mathrm{da}$, com conhecimento na área referente a sistemas de classificação de pacientes. Dessa forma, houve possibilidade de análise da dependência da criança sob diferentes perspectivas, além de valorizar as contribuições tanto do conhecimento acadêmico quanto da prática profissional no cuidado direto ao paciente.

Como critérios de inclusão, foram escolhidos enfermeiros especialistas em pediatria ou com experiência prática mínima de cinco anos nesta área e docentes da área de pediatria ou com estudos voltados a sistemas de classificação de pacientes.

Os juízes receberam instruções específicas, por escrito, sobre o objetivo do estudo e como realizar as avaliações, caso concordassem em participar, por meio de instrumento de registro elaborado para a análise das conceituações propostas. Cada uma das definições de categorias de cuidado deveria ser analisada quanto à concordância e clareza, havendo ainda solicitação de sugestões para melhorar as conceituações.

Para considerar as conceituações válidas, ponderou-se que uma concordância maior que dois terços do grupo de juízes seria representativa; com isso, foi adotado o nível de concordância de $70 \%$ tanto no que se refere à definição quanto à clareza da redação das categorias de cuidado.

O projeto foi encaminhado ao Comitê de Ética e Pesquisa da Faculdade de Ciências Médicas da Universidade Estadual de Campinas e recebeu parecer favorável (no 533/2005).

\section{RESULTADOS}

A primeira versão da conceituação das categorias de cuidado para pacientes pediátricos, constituída por cinco categorias, foi elaborada a partir dos conceitos disponíveis na literatura sobre sistemas de classificação de pacientes ${ }^{16-}$ ${ }^{12)}$ e desenvolvimento infantil(15-17). Cada categoria de cuidado foi descrita segundo as diferentes faixas etárias e está apresentada a seguir:

\section{- Cuidados Mínimos:}

Lactente ou toddler, estável sob o ponto de vista clínico e de enfermagem, acompanhado pela mãe ou responsável, em período integral, com o acompanhante executando atividades de alimentação, higiene e conforto, independente da enfermagem.

Pré-escolar ou escolar estável sob o ponto de vista clínico e de enfermagem, acompanhado por uma pessoa de confiança em período integral, em conjunto com a qual realizam ações de auto-cuidado, independente da enfermagem.

Adolescente estável, sob o ponto de vista clínico e de enfermagem, tendo ou não acompanhante em período integral, colabora com o tratamento e realiza ações de autocuidado sob a supervisão do enfermeiro.

- Cuidados Intermediários:

Lactente ou toddler, estável sob o ponto de vista clínico e de enfermagem, acompanhado pela mãe ou responsável, em período integral, com o acompanhante executando atividades de alimentação, higiene e conforto, mas requer orientações do enfermeiro para essas atividades.

Pré-escolar ou escolar estável sob o ponto de vista clínico e de enfermagem, acompanhado por uma pessoa de confiança em período integral, em conjunto com a qual realizam ações de auto-cuidado, mas requer orientações do enfermeiro para essas atividades.

Adolescente estável, sob o ponto de vista clínico e de enfermagem, tendo ou não acompanhante em período integral, colabora com o tratamento e realiza ações de autocuidado, mas requer orientações do enfermeiro para essas atividades.

- Cuidados de Alta-dependência:

Lactente ou toddler ou pré-escolar ou escolar estável sob o ponto de vista clínico e de enfermagem, portador ou não de alguma patologia crônica, que mesmo com acompanhante em período integral precisa de auxílio nas atividades de alimentação, higiene e conforto e/ou outros cuidados específicos de enfermagem.

Adolescente estável, sob o ponto de vista clínico e de enfermagem, portador ou não de alguma patologia crônica, que independente da presença do acompanhante, não colabora com o tratamento e/ou precisa de auxílio nas atividades de alimentação, higiene e conforto ou outros cuidados específicos de enfermagem.

\section{- Cuidados Semi-Intensivos:}

Paciente pediátrico (RN, lactente, pré-escolar, escolar ou adolescente) sujeito a instabilidade de sinais vitais ou de níveis glicêmicos, sem risco iminente de morte, que, independente de ter um acompanhante, necessita de assistência de enfermagem e médica permanente e especializada. 


\section{- Cuidados Intensivos:}

Paciente pediátrico ( $\mathrm{RN}$, lactente, pré-escolar, escolar ou adolescente) em estado grave, sujeito a instabilidade das funções vitais, com risco iminente de morte, e que necessita de assistência de enfermagem e médica permanente e especializada.

Para a validação de conteúdo das categorias de cuidado, as conceituações propostas foram apreciadas por dez juízas, todas enfermeiras, com idades entre 26 a 48 anos e com tempo de formação entre cinco a 23 anos. Em relação às áreas de atuação profissional, uma delas atua na assistência direta, quatro na gerência de unidades de atendimento pediátrico, duas no ensino de disciplinas de pediatria, duas em ensino e pesquisa na área de pediatria e uma no ensino de administração em enfermagem com pesquisas voltadas a sistemas de classificação de pacientes e gerenciamento em enfermagem. No que concerne à qualificação, três possuem título de especialista, quatro possuem Mestrado e três Doutorado. A primeira avaliação foi realizada por sete juízas e os resultados estão apresentados na Tabela 1.

Tabela 1 - Avaliação das conceituações das categorias de cuidado em pediatria, quanto à concordância e clareza, primeira etapa Campinas - 2006

\begin{tabular}{lcccc}
\hline \multirow{2}{*}{ Categorias de Cuidado } & \multicolumn{2}{c}{ Concordância } & \multicolumn{2}{c}{ Clareza } \\
\cline { 2 - 5 } Cuidados Mínimos & $\mathbf{N}$ & $\mathbf{\%}$ & $\mathbf{N}$ & $\mathbf{\%}$ \\
RN, Lactente & 2 & 29 & 4 & 57 \\
Pré Escolar/Escolar & 3 & 43 & 4 & 57 \\
Adolescente & 5 & 71 & 5 & 71 \\
\hline Intermediários & & & & \\
Lactente/Toddler & 4 & 57 & 3 & 43 \\
Pré Escolar/Escolar & 4 & 57 & 3 & 43 \\
Adolescente & 5 & 71 & 4 & 57 \\
\hline Alta Dependência & & & & \\
Lactente/Toddler/Pré escolar/ & 5 & 71 & 4 & 57 \\
Escolar & 5 & 71 & 4 & 57 \\
Adolescente & 5 & 71 & 5 & 71 \\
\hline Semi intensivos & 5 & 71 & 5 & 71 \\
Intensivos & & & & \\
\hline Nota: (No & 5 & & & \\
\hline
\end{tabular}

Nota: $(\mathrm{N}=7)$

A avaliação da primeira etapa da técnica Delphi resultou em um nível de concordância menor que $70 \%$ quanto às categorias de Cuidados Mínimos e Intermediários para as faixas etárias: RN, lactentes, pré-escolares e escolares; e quanto à clareza de todas as categorias, exceto para Cuidados Semi-intensivos e Intensivos, as quais apresentaram concordância e clareza limítrofe (71\%) com o nível estabelecido para este estudo.

Justificando a necessidade de muitas reformulações na conceituação e a importância do tema, três juízas optaram por não responder ao instrumento de forma completa e se disponibilizaram a um encontro com uma das pesquisadoras para discutir mais aprofundadamente a taxonomia da complexidade de cuidados para pacientes pediátricos.
Diante dos resultados obtidos na primeira etapa da técnica Delphi, as pesquisadoras julgaram conveniente responder positivamente às apreciações dessas juízas. Entre a primeira e segunda etapa, realizaram-se duas reuniões, sendo a primeira com uma juíza e a segunda com as outras duas juízas em forma de oficina de trabalho, na qual foi possível re-elaborar os conceitos os quais são apresentados a seguir:

- Cuidados Mínimos: Paciente pediátrico a partir de 12 anos, com desenvolvimento adequado à idade, estável sob o ponto de vista clínico, realizando todas as ações de autocuidado sob supervisão da enfermagem.

- Cuidados Intermediários: Paciente pediátrico a partir de 7 anos, com desenvolvimento adequado à idade, estável sob o ponto de vista clínico, que necessite de auxílio da enfermagem para seu auto-cuidado e/ou apoio para o enfrentamento da situação de doença e hospitalização.

- Cuidados de Alta-dependência: Paciente pediátrico (de qualquer idade), estável sob o ponto de vista clínico, que dependa da enfermagem para atendimento de suas necessidades orgânicas/físicas, emocionais e sociais.

- Cuidados Semi-Intensivos: Paciente pediátrico (de qualquer idade), instável sob o ponto de vista clínico, sem risco iminente de morte, que necessita de assistência de enfermagem e médica permanente e especializada.

- Cuidados Intensivos: Paciente pediátrico (de qualquer idade), instável sob o ponto de vista clínico, com risco iminente de morte, que necessita de assistência de enfermagem e médica permanente e especializada.

As conceituações reformuladas foram submetidas à apreciação dos demais oito juízes que não participaram da segunda reunião, os quais foram novamente solicitados a avaliar a conceituação e clareza por meio de novo questionário.

Foram enviados oito questionários com retorno de sete $(87,5 \%)$ dos juízes. Destaca-se que todas as categorias de cuidado obtiveram excelente concordância (de 85 a 100\%), tanto no que diz respeito à conceituação, quanto para a clareza, garantido-se a validade do conteúdo das mesmas. Além disto, pode-se considerar que todas elas tiveram $100 \%$ de concordância das duas juízas que participaram da oficina de revisão das conceituações (Tabela 2).

Tabela 2 - Avaliação das conceituações das categorias de cuidado em pediatria, quanto à concordância e clareza, segunda etapa Campinas - 2006

\begin{tabular}{lcccc}
\hline \multirow{2}{*}{ Categorias de Cuidado } & \multicolumn{2}{c}{ Concordância } & \multicolumn{2}{c}{ Clareza } \\
\cline { 2 - 5 } & $\mathbf{N}$ & $\mathbf{\%}$ & $\mathbf{N}$ & $\mathbf{\%}$ \\
\hline Mínimos & 6 & 86 & 6 & 86 \\
Intermediários & 6 & 86 & 6 & 86 \\
Alta dependência & 6 & 86 & 6 & 86 \\
Semi-intensivo & 7 & 100 & 7 & 100 \\
Intensivos & 7 & 100 & 7 & 100 \\
\hline
\end{tabular}

Nota: $(\mathrm{N}=7)$ 


\section{DISCUSSÃO}

$\mathrm{Na}$ literatura internacional|(6,9-11) encontra-se apenas a descrição das propriedades de instrumentos de classificação de pacientes e sua aplicabilidade, no entanto não há conceitos explícitos das categorias de cuidado das mesmas. $\mathrm{Na}$ literatura nacional, os conceitos encontrados de categorias de cuidado para pacientes adultos não são adequados a pacientes pediátricos por não considerarem especificidades da demanda de cuidado para diferentes faixas etárias da clientela, as quais são tão determinantes quanto o grau de complexidade ou severidade da doença pela qual os pacientes pediátricos são hospitalizados ${ }^{(7)}$.

Destaca-se que os juízes concordaram com as categorias de cuidado propostas, que se assemelham às utilizadas para classificar pacientes adultos ${ }^{(7)}$. Entretanto, os juízes discordaram da conceituação segundo a faixa etária para cada categoria de cuidado, justificando que os conceitos dessas categorias são inadequados a pacientes pediátricos por não contemplarem características específicas de desenvolvimento infantil e necessidades de cuidados para cada faixa etária.

Na primeira etapa da avaliação, os juízes colocaram como questões críticas e inaceitáveis o pressuposto de que o acompanhante seria responsável pelos cuidados do paciente internado e a possibilidade de considerar lactentes, pré-escolares e escolares nas categorias de Cuidados Mínimos.

A opinião do grupo de juízes convergiu com a literatura quanto à realização de cuidados pelos familiares, uma vez que podem executar muitos dos cuidados à criança, desde que queiram e sintam-se hábeis, mas, durante todo o período de internação, entende-se que os cuidados com o paciente são de integral responsabilidade da enfermagem.

Considera-se acertada a decisão de conceituar a categoria de Alta dependência, pois essa categoria está estreitamente ligada às características inerentes aos pacientes pediátricos e, independente da gravidade e complexidade da doença pela qual uma criança é hospitalizada, a mesma depende de adultos em atividades básicas do dia a dia, além de necessitar de supervisão contínua de modo a garantir sua segurança(17).

A escolha da técnica Delphi para a avaliação das categorias de cuidado ocorreu devido à sua flexibilidade, que permite a realização de tantas etapas de apreciação quanto forem necessárias até alcançar-se o nível de concordância pretendido. A vantagem dessa técnica é alcançar um bom número de respondentes de diferentes atuações profissionais, como assistência direta, gerência, ensino e pesquisa, ainda que sejam de localidades bem distantes do pesquisador, tornando mais ampla a apreciação( ${ }^{(13-14)}$.

Entretanto, a incorporação das mudanças sugeridas à distância não é tarefa simples, o que pode gerar a necessidade de vários retornos do modelo aos juízes, até que se atinja um elevado grau de concordância. Desta forma, ao obter baixo nível de concordância e clareza para as categorias de cuidado após a primeira fase da técnica Delphi verificou-se que devido à importância do tema, a ausência de encontro presencial entre os juízes impossibilitaria consensuar e conceituar as categorias de cuidado em pediatria.

A realização do encontro presencial com duas juízas foi um fator favorável. Avalia-se que o debate direto entre pessoas com diferentes inserções e experiências profissionais possibilitou a elaboração de uma proposta que se mostrou bem aceita pelos juízes que fizeram a avaliação à distância. Tendo por base esta experiência, pode-se sugerir o trabalho em grupo como estratégia metodológica para o delineamento do modelo a ser analisado, antecedendo a aplicação da Técnica Delphi.

A validade das conceituações foi garantida após dois momentos de contato entre a pesquisadora e cada juiz, de forma que após a reformulação obteve-se excelente nível de concordância do grupo para as novas conceituações (entre 85 e $100 \%)$.

O delineamento de cinco categorias de cuidados em pediatria viabiliza a realização de novos estudos que possibilitem estimar o tempo gasto pela equipe de enfermagem no atendimento das necessidades dos pacientes pediátricos, subsidiando a tomada de decisão acerca da adequação do quadro de pessoal, tanto nos aspectos quantitativos, quanto nos qualitativos.

\section{CONCLUSÃO}

O estudo viabilizou uma proposta de categorização dos pacientes pediátricos relativamente simples e, portanto, com potencial de utilização para fundamentar o dimensionamento de pessoal em enfermagem pediátrica.

Foram conceituadas e validadas cinco Categorias de Cuidado em Pediatria: Cuidados mínimos, Cuidados intermediários, Cuidados de alta dependência, Cuidados semi-intensivos e Cuidados intensivos.

Destaca-se que o processo de validação de conteúdo das conceituações, por meio da Técnica Delphi, bem como os encontros presenciais com juízes envolvidos em ensino, pesquisa, na assistência direta e gerência de unidades pediátricas, com diferentes competências para avaliar o método de classificação de pacientes em pediatria, subsidiou modificações fundamentais na definição das categorias de cuidado propostas, contemplando a realidade assistencial e administrativa das unidades de internação pediátricas.

A classificação de pacientes em categorias de cuidado fornece subsídios para o dimensionamento de pessoal de enfermagem na área de enfermagem pediátrica, no entanto, faz-se necessário identificar as horas médias de assistência de enfermagem para cada categoria de cuidado em pediatria. 


\section{REFERÊNCIAS}

1. Pinheiro RS, Escosteguy CC. Epidemiologia e serviços de Saúde. In: Medronho RA, Carvalho DM, Bloch KV, Luiz RR, Werneck GL. Epidemiologia. São Paulo: Atheneu; 2003. p. 361-9.

2. Campbell SM, Roland MO, Buetow SA. Defining quality of care. Soc Sci Med. 2000; 51(11):1611-25.

3. Fugulin FMT. Dimensionamento de pessoal de enfermagem: avaliação do quadro de pessoal das unidades de internação de um hospital de ensino [tese doutorado]. São Paulo: Escola de Enfermagem, Universidade de São Paulo; 2002.

4. Tanos MAA, Massarollo MCKB, Gaidzinski RR. Dimensionamento de pessoal de enfermagem em uma unidade especializada em transplante de fígado: comparação do real com o preconizado. Rev Esc Enferm USP. 2000;34(4):376-82.

5. Gaidzinski RR, Fugulin FMT, Castilho,V. Dimensionamento de pessoal de enfermagem em instituições de saúde. In: Kurcgant $P$, coordenadora. Gerenciamento em enfermagem. Rio de Janeiro: Guanabara Koogan; 2005. p.125-37.

6. Huber D. Staffing and scheduling. In: Huber D. Leadership and nursing care management. Philadelphia: Sauders; 2000. p. 573-90.

7. Fugulin FMT, Silva SHS, Shimizu HE, Campos FPF. Implantação do Sistema de Classificação de Pacientes na Unidade de Clínica Médica do Hospital Universitário da USP. Rev Med HU-USP. 1994;4(1/2):63-8.

8. Conselho Federal de Enfermagem. Resolução COFEN 293/2004. Parâmetros para o Dimensionamento do Quadro de Profissionais de Enfermagem nas Unidades Assistenciais das Instituições de Saúde e Assemelhados [Internet]. Brasília; 2004 [citado 2009 fev. 9]. Disponível em: http://www.saude.mg.gov.br/ atos_normativos/legislacao-sanitaria/estabelecimentos-desaude/exercicio-profissional/res_293.pdf
9. Rainio AK, Ohinmaa AE. Assessment of nursing management and utilization of nursing resourcer with the RAFAELA patient classification system-case study from the general wards of one central hospital. J Clin Nurs. 2005;14(6):674-84.

10. Giovannetti P. Understanding patient classification systems. J Nurs Adm. 1979;9(2):4-9.

11. De Groot HA. Patient classification system evaluation. Part 1: Essential system elements. J Nurs Adm. 1989;19(6):30-5.

12. Lobiondo-Wood G, Haber J. Pesquisa em enfermagem: métodos, avaliação crítica e utilização. 4ạ ed. Rio de Janeiro: Guanabara Koogan; 2001.

13. Goodman CM. The Delphi technique: a critique. J Adv Nurs. 1987;12(6):729-34.

14. Williams PL, Webb C. The Delphi technique: a methodological discussion. J Adv Nurs. 1994;19(1):180-6.

15. Veríssimo MLOR, Sigaud CHS. O acompanhamento do crescimento e desenvolvimento da criança. In: Sigaud CHS, Veríssimo MLOR. Enfermagem pediátrica: o cuidado de enfermagem à criança e ao adolescente. São Paulo: EPU; 1996. p.137-62.

16. Wong DL. Enfermagem pediátrica: elementos essenciais à intervenção efetiva. Rio de Janeiro: Guanabara Koogan; 1997. p. 73-92.

17. Brazelton TB, Greenspan SI. As necessidades essenciais das crianças. Porto Alegre: Artmed; 2002. 